Bull. Korean Math. Soc. 48 (2011), No. 3, pp. 491-503

DOI 10.4134/BKMS.2011.48.3.491

\title{
FUZZY STABILITY OF A CUBIC-QUARTIC FUNCTIONAL EQUATION: A FIXED POINT APPROACH
}

\author{
Sun-Young Jang, Choonkil Park, And Dong Yun Shin
}

ABstract. Using the fixed point method, we prove the generalized HyersUlam stability of the following cubic-quartic functional equation

$(0.1)$

$$
\begin{aligned}
f(2 x+y)+f(2 x-y)= & 3 f(x+y)+f(-x-y)+3 f(x-y)+f(y-x) \\
& +18 f(x)+6 f(-x)-3 f(y)-3 f(-y)
\end{aligned}
$$

in fuzzy Banach spaces.

\section{Introduction and preliminaries}

The theory of fuzzy space has much progressed as the theory of randomness has developed. Some mathematicians have defined fuzzy norms on a vector space from various points of view [2, 11, 20, 24, 37]. Following Cheng and Mordeson [7], Bag and Samanta [2] gave an idea of fuzzy norm in such a manner that the corresponding fuzzy metric is of Kramosil and Michalek type [19] and investigated some properties of fuzzy normed spaces [3].

We use the definition of fuzzy normed spaces given in $[2,24,25]$ to investigate a fuzzy version of the generalized Hyers-Ulam stability for the functional equation (0.1) in the fuzzy normed vector space setting.

Definition $1.1([2,24,25,26])$. Let $X$ be a real vector space. A function $N: X \times \mathbb{R} \rightarrow[0,1]$ is called a fuzzy norm on $X$ if for all $x, y \in X$ and all $s, t \in \mathbb{R}$,

$\left(N_{1}\right) N(x, t)=0$ for $t \leq 0$

$\left(N_{2}\right) x=0$ if and only if $N(x, t)=1$ for all $t>0$;

$\left(N_{3}\right) N(c x, t)=N\left(x, \frac{t}{|c|}\right)$ if $c \neq 0$

$\left(N_{4}\right) N(x+y, s+t) \geq \min \{N(x, s), N(y, t)\}$;

Received September 2, 2009; Revised April 30, 2011.

2010 Mathematics Subject Classification. Primary 46S40, 39B72; Secondary 39B52, 46S50, 26E50, 47H10.

Key words and phrases. fuzzy Banach space, fixed point, generalized Hyers-Ulam stability, quartic mapping, cubic mapping.

The first author was supported by University of Ulsan, 2009 Research Fund and had written during visiting the Research Institute of Mathematics, Seoul National University. 
$\left(N_{5}\right) N(x, \cdot)$ is a non-decreasing function of $\mathbb{R}$ and $\lim _{t \rightarrow \infty} N(x, t)=1$;

$\left(N_{6}\right)$ for $x \neq 0, N(x, \cdot)$ is continuous on $\mathbb{R}$.

The pair $(X, N)$ is called a fuzzy normed vector space.

The properties of fuzzy normed vector spaces and examples of fuzzy norms are given in [24, 23].

Definition $1.2([2,24,25,26])$. Let $(X, N)$ be a fuzzy normed vector space. A sequence $\left\{x_{n}\right\}$ in $X$ is said to be convergent or converge if there exists an $x \in X$ such that $\lim _{n \rightarrow \infty} N\left(x_{n}-x, t\right)=1$ for all $t>0$. In this case, $x$ is called the limit of the sequence $\left\{x_{n}\right\}$ and we denote it by $N-\lim _{n \rightarrow \infty} x_{n}=x$.

Definition $1.3([2,24,25])$. Let $(X, N)$ be a fuzzy normed vector space. A sequence $\left\{x_{n}\right\}$ in $X$ is called Cauchy if for each $\varepsilon>0$ and each $t>0$ there exists an $n_{0} \in \mathbb{N}$ such that for all $n \geq n_{0}$ and all $p>0$, we have $N\left(x_{n+p}-x_{n}, t\right)>1-\varepsilon$.

It is well-known that every convergent sequence in a fuzzy normed vector space is Cauchy. If each Cauchy sequence is convergent, then the fuzzy norm is said to be complete and the fuzzy normed vector space is called a fuzzy Banach space.

We say that a mapping $f: X \rightarrow Y$ between fuzzy normed vector spaces $X$ and $Y$ is continuous at a point $x_{0} \in X$ if for each sequence $\left\{x_{n}\right\}$ converging to $x_{0}$ in $X$, the sequence $\left\{f\left(x_{n}\right)\right\}$ converges to $f\left(x_{0}\right)$. If $f: X \rightarrow Y$ is continuous at each $x \in X, f: X \rightarrow Y$ is said to be continuous on $X$ (see [3]).

The stability problem of functional equations originated from a question of Ulam [36] concerning the stability of group homomorphisms. Hyers [13] gave a first affirmative partial answer to the question of Ulam for Banach spaces. Hyers' Theorem was generalized by Aoki [1] for additive mappings and by Th. M. Rassias [33] for linear mappings by considering an unbounded Cauchy difference. The paper of Th. M. Rassias [33] has provided a lot of influence in the development of what we call generalized Hyers-Ulam stability or as HyersUlam-Rassias stability of functional equations. A generalization of the Th. M. Rassias theorem was obtained by Găvruta [12] by replacing the unbounded Cauchy difference by a general control function in the spirit of Th. M. Rassias' approach.

The functional equation

$$
f(x+y)+f(x-y)=2 f(x)+2 f(y)
$$

is called a quadratic functional equation. In particular, every solution of the quadratic functional equation is said to be a quadratic function. A generalized Hyers-Ulam stability problem for the quadratic functional equation was proved by Skof [35] for mappings $f: X \rightarrow Y$, where $X$ is a normed space and $Y$ is a Banach space. Cholewa [8] noticed that the theorem of Skof is still true if the relevant domain $X$ is replaced by an Abelian group. Czerwik [9] proved the generalized Hyers-Ulam stability of the quadratic functional equation. The 
stability problems of several functional equations have been extensively investigated by a number of authors and there are many interesting results concerning this problem (see [14, 17], [29]-[31], [34]).

In [16], Jun and Kim considered the following cubic functional equation

$$
f(2 x+y)+f(2 x-y)=2 f(x+y)+2 f(x-y)+12 f(x) .
$$

It is easy to show that the function $f(x)=x^{3}$ satisfies the functional equation (1.1), which is called a cubic functional equation and every solution of the cubic functional equation is said to be a cubic mapping.

In [21], Lee et al. considered the following quartic functional equation

$$
f(2 x+y)+f(2 x-y)=4 f(x+y)+4 f(x-y)+24 f(x)-6 f(y) .
$$

It is easy to show that the function $f(x)=x^{4}$ satisfies the functional equation (1.2), which is called a quartic functional equation and every solution of the quartic functional equation is said to be a quartic mapping.

The functional equation (0.1) is a cubic-quartic functional equation because (0.1) is quartic when $f(x)$ is a even function and (0.1) is cubic when $f(x)$ is a odd function.

We recall a fundamental result in fixed point theory.

Let $X$ be a set. A function $d: X \times X \rightarrow[0, \infty]$ is called a generalized metric on $X$ if $d$ satisfies

(1) $d(x, y)=0$ if and only if $x=y$;

(2) $d(x, y)=d(y, x)$ for all $x, y \in X$;

(3) $d(x, z) \leq d(x, y)+d(y, z)$ for all $x, y, z \in X$.

Theorem 1.4 ([4, 10]). Let $(X, d)$ be a complete generalized metric space and let $J: X \rightarrow X$ be a strictly contractive mapping with Lipschitz constant $L<1$. Then for each given element $x \in X$, either

$$
d\left(J^{n} x, J^{n+1} x\right)=\infty
$$

for all nonnegative integers $n$ or there exists a positive integer $n_{0}$ such that

(1) $d\left(J^{n} x, J^{n+1} x\right)<\infty, \quad \forall n \geq n_{0}$;

(2) the sequence $\left\{J^{n} x\right\}$ converges to a fixed point $y^{*}$ of $J$;

(3) $y^{*}$ is the unique fixed point of $J$ in the set $Y=\left\{y \in X \mid d\left(J^{n_{0}} x, y\right)<\infty\right\}$;

(4) $d\left(y, y^{*}\right) \leq \frac{1}{1-L} d(y, J y)$ for all $y \in Y$.

In 1996, G. Isac and Th. M. Rassias [15] were the first to provide applications of new fixed point theorems for the proof of stability theory of functional equations. By using fixed point methods, the stability problems of several functional equations have been extensively investigated by a number of authors (see $[5,6,23,27,28,32])$.

This paper is organized as follows: In Section 2, we prove the generalized Hyers-Ulam stability of the cubic-quartic functional equation (0.1) in fuzzy Banach spaces for an odd mapping case. In Section 3, we prove the generalized Hyers-Ulam stability of the cubic-quartic functional equation (0.1) in fuzzy Banach spaces for an even mapping case. 
Throughout this paper, assume that $X$ is a vector space and that $(Y, N)$ is a fuzzy Banach space.

\section{Generalized Hyers-Ulam stability of the functional equation (0.1): an odd mapping case}

One can easily show that an even mapping $f: X \rightarrow Y$ satisfies (0.1) if and only if the even mapping $f: X \rightarrow Y$ is a quartic mapping, i.e.,

$$
f(2 x+y)+f(2 x-y)=4 f(x+y)+4 f(x-y)+24 f(x)-6 f(y),
$$

and that an odd mapping $f: X \rightarrow Y$ satisfies (0.1) if and only if the odd mapping $f: X \rightarrow Y$ is a cubic mapping, i.e.,

$$
f(2 x+y)+f(2 x-y)=2 f(x+y)+2 f(x-y)+12 f(x) .
$$

For a given mapping $f: X \rightarrow Y$, we define

$$
\begin{aligned}
D f(x, y):= & f(2 x+y)+f(2 x-y)-3 f(x+y)-f(-x-y)-3 f(x-y) \\
& -f(y-x)-18 f(x)-6 f(-x)+3 f(y)+3 f(-y)
\end{aligned}
$$

for all $x, y \in X$.

Using the fixed point method, we prove the generalized Hyers-Ulam stability of the functional equation $D f(x, y)=0$ in fuzzy Banach spaces: an odd case.

Theorem 2.1. Let $\varphi: X^{2} \rightarrow[0, \infty)$ be a function such that there exists an $L<1$ with

$$
\varphi(x, y) \leq \frac{L}{8} \varphi(2 x, 2 y)
$$

for all $x, y \in X$. Let $f: X \rightarrow Y$ be an odd mapping satisfying

$$
N(D f(x, y), t) \geq \frac{t}{t+\varphi(x, y)}
$$

for all $x, y \in X$ and all $t>0$. Then $C(x):=N$ - $\lim _{n \rightarrow \infty} 8^{n} f\left(\frac{x}{2^{n}}\right)$ exists for each $x \in X$ and defines a cubic mapping $C: X \rightarrow Y$ such that

$$
N(f(x)-C(x), t) \geq \frac{(16-16 L) t}{(16-16 L) t+L \varphi(x, 0)}
$$

for all $x \in X$ and all $t>0$.

Proof. Letting $y=0$ in (2.1), we get

$$
N(2 f(2 x)-16 f(x), t) \geq \frac{t}{t+\varphi(x, 0)}
$$

for all $x \in X$.

Consider the set

$$
S:=\{g: X \rightarrow Y\}
$$

and introduce the generalized metric on $S$ :

$$
d(g, h)=\inf \left\{\mu \in \mathbb{R}_{+}: N(g(x)-h(x), \mu t) \geq \frac{t}{t+\varphi(x, 0)}, \forall x \in X, \forall t>0\right\},
$$


where, as usual, $\inf \phi=+\infty$. It is easy to show that $(S, d)$ is complete (See the proof of [22, Lemma 2.1]).

Now we consider the linear mapping $J: S \rightarrow S$ such that

$$
J g(x):=8 g\left(\frac{x}{2}\right)
$$

for all $x \in X$.

Let $g, h \in S$ be given such that $d(g, h)=\varepsilon$. Then

$$
N(g(x)-h(x), \varepsilon t) \geq \frac{t}{t+\varphi(x, 0)}
$$

for all $x \in X$ and all $t>0$. Hence

$$
\begin{aligned}
N(J g(x)-J h(x), L \varepsilon t) & =N\left(8 g\left(\frac{x}{2}\right)-8 h\left(\frac{x}{2}\right), L \varepsilon t\right) \\
& =N\left(g\left(\frac{x}{2}\right)-h\left(\frac{x}{2}\right), \frac{L}{8} \varepsilon t\right) \\
& \geq \frac{\frac{L t}{8}}{\frac{L t}{8}+\varphi\left(\frac{x}{2}, 0\right)} \\
& \geq \frac{\frac{L t}{8}}{\frac{L t}{8}+\frac{L}{8} \varphi(x, 0)} \\
& =\frac{t}{t+\varphi(x, 0)}
\end{aligned}
$$

for all $x \in X$ and all $t>0$. So $d(g, h)=\varepsilon$ implies that $d(J g, J h) \leq L \varepsilon$. This means that

for all $g, h \in S$.

$$
d(J g, J h) \leq L d(g, h)
$$

It follows from (2.3) that

$$
N\left(f(x)-8 f\left(\frac{x}{2}\right), \frac{L}{16} t\right) \geq \frac{t}{t+\varphi(x, 0)}
$$

for all $x \in X$ and all $t>0$. So $d(f, J f) \leq \frac{L}{16}$.

By Theorem 1.4, there exists a mapping $C: X \rightarrow Y$ satisfying the following:

(1) $C$ is a fixed point of $J$, i.e.,

$$
C\left(\frac{x}{2}\right)=\frac{1}{8} C(x)
$$

for all $x \in X$. Since $f: X \rightarrow Y$ is odd, $C: X \rightarrow Y$ is an odd mapping. The mapping $C$ is a unique fixed point of $J$ in the set

$$
M=\{g \in S: d(f, g)<\infty\} .
$$

This implies that $C$ is a unique mapping satisfying (2.4) such that there exists a $\mu \in(0, \infty)$ satisfying

$$
N(f(x)-C(x), \mu t) \geq \frac{t}{t+\varphi(x, 0)}
$$


for all $x \in X$;

(2) $d\left(J^{n} f, C\right) \rightarrow 0$ as $n \rightarrow \infty$. This implies the equality

$$
N-\lim _{n \rightarrow \infty} 8^{n} f\left(\frac{x}{2^{n}}\right)=C(x)
$$

for all $x \in X$;

(3) $d(f, C) \leq \frac{1}{1-L} d(f, J f)$, which implies the inequality

$$
d(f, C) \leq \frac{L}{16-16 L} .
$$

This implies that the inequality (2.2) holds.

By $(2.1)$,

$$
N\left(8^{n} D f\left(\frac{x}{2^{n}}, \frac{y}{2^{n}}\right), 8^{n} t\right) \geq \frac{t}{t+\varphi\left(\frac{x}{2^{n}}, \frac{y}{2^{n}}\right)}
$$

for all $x, y \in X$, all $t>0$ and all $n \in \mathbb{N}$. So

$$
N\left(8^{n} D f\left(\frac{x}{2^{n}}, \frac{y}{2^{n}}\right), t\right) \geq \frac{\frac{t}{8^{n}}}{\frac{t}{8^{n}}+\frac{L^{n}}{8^{n}} \varphi(x, y)}
$$

for all $x, y \in X$, all $t>0$ and all $n \in \mathbb{N}$. Since $\lim _{n \rightarrow \infty} \frac{\frac{t}{8^{n}}}{\frac{t}{8^{n}}+\frac{L^{n}}{8^{n}} \varphi(x, y)}=1$ for all $x, y \in X$ and all $t>0$,

$$
N(D C(x, y), t)=1
$$

for all $x, y \in X$ and all $t>0$. Thus the mapping $C: X \rightarrow Y$ is cubic, as desired.

Corollary 2.2. Let $\theta \geq 0$ and let $p$ be a real number and $p>3$. Let $X$ be a normed vector space with norm $\|\cdot\|$. Let $f: X \rightarrow Y$ be an odd mapping satisfying

$$
N(D f(x, y), t) \geq \frac{t}{t+\theta\left(\|x\|^{p}+\|y\|^{p}\right)}
$$

for all $x, y \in X$ and all $t>0$. Then $C(x):=N-\lim _{n \rightarrow \infty} 8^{n} f\left(\frac{x}{2^{n}}\right)$ exists for each $x \in X$ and defines a cubic mapping $C: X \rightarrow Y$ such that

$$
N(f(x)-C(x), t) \geq \frac{2\left(2^{p}-8\right) t}{2\left(2^{p}-8\right) t+\theta\|x\|^{p}}
$$

for all $x \in X$ and all $t>0$.

Proof. The proof follows from Theorem 2.1 by taking

$$
\varphi(x, y):=\theta\left(\|x\|^{p}+\|y\|^{p}\right)
$$

for all $x, y \in X$. Then we can choose $L=2^{3-p}$ and we get the desired result.

Theorem 2.3. Let $\varphi: X^{2} \rightarrow[0, \infty)$ be a function such that there exists an $L<1$ with

$$
\varphi(x, y) \leq 8 L \varphi\left(\frac{x}{2}, \frac{y}{2}\right)
$$


for all $x, y \in X$. Let $f: X \rightarrow Y$ be an odd mapping satisfying (2.1). Then $C(x):=N-\lim _{n \rightarrow \infty} \frac{1}{8^{n}} f\left(2^{n} x\right)$ exists for each $x \in X$ and defines a cubic mapping $C: X \rightarrow Y$ such that

$$
N(f(x)-C(x), t) \geq \frac{(16-16 L) t}{(16-16 L) t+\varphi(x, 0)}
$$

for all $x \in X$ and all $t>0$.

Proof. Let $(S, d)$ be the generalized metric space defined in the proof of Theorem 2.1 .

Consider the linear mapping $J: S \rightarrow S$ such that

$$
J g(x):=\frac{1}{8} g(2 x)
$$

for all $x \in X$.

Let $g, h \in S$ be given such that $d(g, h)=\varepsilon$. Then

$$
N(g(x)-h(x), \varepsilon t) \geq \frac{t}{t+\varphi(x, 0)}
$$

for all $x \in X$ and all $t>0$. Hence

$$
\begin{aligned}
N(J g(x)-J h(x), L \varepsilon t) & =N\left(\frac{1}{8} g(2 x)-\frac{1}{8} h(2 x), L \varepsilon t\right) \\
& =N(g(2 x)-h(2 x), 8 L \varepsilon t) \\
& \geq \frac{8 L t}{8 L t+\varphi(2 x, 0)} \\
& \geq \frac{8 L t}{8 L t+8 L \varphi(x, 0)} \\
& =\frac{t}{t+\varphi(x, 0)}
\end{aligned}
$$

for all $x \in X$ and all $t>0$. So $d(g, h)=\varepsilon$ implies that $d(J g, J h) \leq L \varepsilon$. This means that

$$
d(J g, J h) \leq L d(g, h)
$$

for all $g, h \in S$.

It follows from (2.3) that

$$
N\left(f(x)-\frac{1}{8} f(2 x), \frac{1}{16} t\right) \geq \frac{t}{t+\varphi(x, 0)}
$$

for all $x \in X$ and all $t>0$. So $d(f, J f) \leq \frac{1}{16}$.

By Theorem 1.4, there exists a mapping $C: X \rightarrow Y$ satisfying the following:

(1) $C$ is a fixed point of $J$, i.e.,

$$
C(2 x)=8 C(x)
$$


for all $x \in X$. Since $f: X \rightarrow Y$ is odd, $C: X \rightarrow Y$ is an odd mapping. The mapping $C$ is a unique fixed point of $J$ in the set

$$
M=\{g \in S: d(f, g)<\infty\} .
$$

This implies that $C$ is a unique mapping satisfying (2.7) such that there exists a $\mu \in(0, \infty)$ satisfying

$$
N(f(x)-C(x), \mu t) \geq \frac{t}{t+\varphi(x, 0)}
$$

for all $x \in X$;

(2) $d\left(J^{n} f, C\right) \rightarrow 0$ as $n \rightarrow \infty$. This implies the equality

$$
N-\lim _{n \rightarrow \infty} \frac{1}{8^{n}} f\left(2^{n} x\right)=C(x)
$$

for all $x \in X$;

(3) $d(f, C) \leq \frac{1}{1-L} d(f, J f)$, which implies the inequality

$$
d(f, C) \leq \frac{1}{16-16 L} .
$$

This implies that the inequality (2.6) holds.

The rest of the proof is similar to the proof of Theorem 2.1.

Corollary 2.4. Let $\theta \geq 0$ and let $p$ be a real number and $0<p<3$. Let $X$ be a normed vector space with norm $\|\cdot\|$. Let $f: X \rightarrow Y$ be an odd mapping satisfying (2.5). Then $C(x):=N-\lim _{n \rightarrow \infty} \frac{1}{8^{n}} f\left(2^{n} x\right)$ exists for each $x \in X$ and defines a cubic mapping $C: X \rightarrow Y$ such that

$$
N(f(x)-C(x), t) \geq \frac{2\left(8-2^{p}\right) t}{2\left(8-2^{p}\right) t+\theta\|x\|^{p}}
$$

for all $x \in X$ and all $t>0$.

Proof. The proof follows from Theorem 2.3 by taking

$$
\varphi(x, y):=\theta\left(\|x\|^{p}+\|y\|^{p}\right)
$$

for all $x, y \in X$. Then we can choose $L=2^{p-3}$ and we get the desired result.

\section{Generalized Hyers-Ulam stability of the functional equation (0.1): an even mapping case}

Using the fixed point method, we prove the generalized Hyers-Ulam stability of the functional equation $D f(x, y)=0$ in fuzzy Banach spaces: an even case.

Theorem 3.1. Let $\varphi: X^{2} \rightarrow[0, \infty)$ be a function such that there exists an $L<1$ with

$$
\varphi(x, y) \leq \frac{L}{16} \varphi(2 x, 2 y)
$$


for all $x, y \in X$. Let $f: X \rightarrow Y$ be an even mapping satisfying $f(0)=0$ and (2.1). Then $Q(x):=N$ - $\lim _{n \rightarrow \infty} 16^{n} f\left(\frac{x}{2^{n}}\right)$ exists for each $x \in X$ and defines $a$ quartic mapping $Q: X \rightarrow Y$ such that

$$
N(f(x)-Q(x), t) \geq \frac{(32-32 L) t}{(32-32 L) t+L \varphi(x, 0)}
$$

for all $x \in X$ and all $t>0$.

Proof. Letting $y=0$ in (2.1), we get

$$
N(2 f(2 x)-32 f(x), t) \geq \frac{t}{t+\varphi(x, 0)}
$$

for all $x \in X$.

Let $(S, d)$ be the generalized metric space defined in the proof of Theorem 2.1 .

Now we consider the linear mapping $J: S \rightarrow S$ such that

$$
J g(x):=16 g\left(\frac{x}{2}\right)
$$

for all $x \in X$.

Let $g, h \in S$ be given such that $d(g, h)=\varepsilon$. Then

$$
N(g(x)-h(x), \varepsilon t) \geq \frac{t}{t+\varphi(x, 0)}
$$

for all $x \in X$ and all $t>0$. Hence

$$
\begin{aligned}
N(J g(x)-J h(x), L \varepsilon t) & =N\left(16 g\left(\frac{x}{2}\right)-16 h\left(\frac{x}{2}\right), L \varepsilon t\right) \\
& =N\left(g\left(\frac{x}{2}\right)-h\left(\frac{x}{2}\right), \frac{L}{16} \varepsilon t\right) \\
& \geq \frac{\frac{L t}{16}}{\frac{L t}{16}+\varphi\left(\frac{x}{2}, 0\right)} \\
& \geq \frac{\frac{L t}{16}}{\frac{L t}{16}+\frac{L}{16} \varphi(x, 0)} \\
& =\frac{t}{t+\varphi(x, 0)}
\end{aligned}
$$

for all $x \in X$ and all $t>0$. So $d(g, h)=\varepsilon$ implies that $d(J g, J h) \leq L \varepsilon$. This means that

$$
d(J g, J h) \leq L d(g, h)
$$

for all $g, h \in S$.

It follows from (3.2) that

$$
N\left(f(x)-16 f\left(\frac{x}{2}\right), \frac{L}{32} t\right) \geq \frac{t}{t+\varphi(x, 0)}
$$

for all $x \in X$ and all $t>0$. So $d(f, J f) \leq \frac{L}{32}$.

By Theorem 1.4, there exists a mapping $Q: X \rightarrow Y$ satisfying the following: 
(1) $Q$ is a fixed point of $J$, i.e.

$$
Q\left(\frac{x}{2}\right)=\frac{1}{16} Q(x)
$$

for all $x \in X$. Since $f: X \rightarrow Y$ is even, $Q: X \rightarrow Y$ is an even mapping. The mapping $Q$ is a unique fixed point of $J$ in the set

$$
M=\{g \in S: d(f, g)<\infty\} .
$$

This implies that $Q$ is a unique mapping satisfying (3.3) such that there exists a $\mu \in(0, \infty)$ satisfying

$$
N(f(x)-Q(x), \mu t) \geq \frac{t}{t+\varphi(x, 0)}
$$

for all $x \in X$;

(2) $d\left(J^{n} f, Q\right) \rightarrow 0$ as $n \rightarrow \infty$. This implies the equality

$$
N-\lim _{n \rightarrow \infty} 16^{n} f\left(\frac{x}{2^{n}}\right)=Q(x)
$$

for all $x \in X$;

(3) $d(f, Q) \leq \frac{1}{1-L} d(f, J f)$, which implies the inequality

$$
d(f, Q) \leq \frac{L}{32-32 L} .
$$

This implies that the inequality (3.1) holds.

The rest of the proof is similar to the proof of Theorem 2.1.

Corollary 3.2. Let $\theta \geq 0$ and let $p$ be a real number and $p>4$. Let $X$ be a normed vector space with norm $\|\cdot\|$. Let $f: X \rightarrow Y$ be an even mapping satisfying $f(0)=0$ and (2.5). Then $Q(x):=N-\lim _{n \rightarrow \infty} 16^{n} f\left(\frac{x}{2^{n}}\right)$ exists for each $x \in X$ and defines a quartic mapping $Q: X \rightarrow Y$ such that

$$
N(f(x)-Q(x), t) \geq \frac{2\left(2^{p}-16\right) t}{2\left(2^{p}-16\right) t+\theta\|x\|^{p}}
$$

for all $x \in X$ and all $t>0$.

Proof. The proof follows from Theorem 3.1 by taking

$$
\varphi(x, y):=\theta\left(\|x\|^{p}+\|y\|^{p}\right)
$$

for all $x, y \in X$. Then we can choose $L=2^{4-p}$ and we get the desired result.

Similarly, we can obtain the following. We will omit the proof.

Theorem 3.3. Let $\varphi: X^{2} \rightarrow[0, \infty)$ be a function such that there exists an $L<1$ with

$$
\varphi(x, y) \leq 16 L \varphi\left(\frac{x}{2}, \frac{y}{2}\right)
$$


for all $x, y \in X$. Let $f: X \rightarrow Y$ be an even mapping satisfying $f(0)=0$ and (2.1). Then $Q(x):=N-\lim _{n \rightarrow \infty} \frac{1}{16^{n}} f\left(2^{n} x\right)$ exists for each $x \in X$ and defines a quartic mapping $Q: X \rightarrow Y$ such that

$$
N(f(x)-Q(x), t) \geq \frac{(32-32 L) t}{(32-32 L) t+\varphi(x, 0)}
$$

for all $x \in X$ and all $t>0$.

Corollary 3.4. Let $\theta \geq 0$ and let $p$ be a real number and $0<p<4$. Let $X$ be a normed vector space with norm $\|\cdot\|$. Let $f: X \rightarrow Y$ be an even mapping satisfying $f(0)=0$ and (2.5). Then $Q(x):=N-\lim _{n \rightarrow \infty} \frac{1}{16^{n}} f\left(2^{n} x\right)$ exists for each $x \in X$ and defines a quartic mapping $Q: X \rightarrow Y$ such that

$$
N(f(x)-Q(x), t) \geq \frac{2\left(16-2^{p}\right) t}{2\left(16-2^{p}\right) t+\theta\|x\|^{p}}
$$

for all $x \in X$ and all $t>0$.

Proof. The proof follows from Theorem 3.3 by taking

$$
\varphi(x, y):=\theta\left(\|x\|^{p}+\|y\|^{p}\right)
$$

for all $x, y \in X$. Then we can choose $L=2^{p-4}$ and we get the desired result.

\section{References}

[1] T. Aoki, On the stability of the linear transformation in Banach spaces, J. Math. Soc. Japan 2 (1950), 64-66.

[2] T. Bag and S. K. Samanta, Finite dimensional fuzzy normed linear spaces, J. Fuzzy Math. 11 (2003), no. 3, 687-705.

[3] _ Fuzzy bounded linear operators, Fuzzy Sets and Systems 151 (2005), no. 3, $513-547$.

[4] L. Cădariu and V. Radu, Fixed points and the stability of Jensen's functional equation, J. Inequal. Pure Appl. Math. 4 (2003), no. 1, Art. 4, 7 pp.

[5] - On the stability of the Cauchy functional equation: a fixed point approach, Iteration theory (ECIT '02), 43-52, Grazer Math. Ber., 346, Karl-Franzens-Univ. Graz, Graz, 2004.

[6] _ Fixed point methods for the generalized stability of functional equations in a single variable, Fixed Point Theory and Applications 2008 (2008), Art. ID 749392.

[7] S. C. Cheng and J. M. Mordeson, Fuzzy linear operators and fuzzy normed linear spaces, Bull. Calcutta Math. Soc. 86 (1994), no. 5, 429-436.

[8] P. W. Cholewa, Remarks on the stability of functional equations, Aequationes Math. 27 (1984), no. 1-2, 76-86.

[9] S. Czerwik, On the stability of the quadratic mapping in normed spaces, Abh. Math. Sem. Univ. Hamburg 62 (1992), 59-64.

[10] J. Diaz and B. Margolis, A fixed point theorem of the alternative for contractions on a generalized complete metric space, Bull. Amer. Math. Soc. 74 (1968), 305-309.

[11] C. Felbin, Finite dimensional fuzzy normed linear spaces, Fuzzy Sets and Systems 48 (1992), no. 2, 239-248.

[12] P. Găvruta, A generalization of the Hyers-Ulam-Rassias stability of approximately additive mappings, J. Math. Anal. Appl. 184 (1994), no. 3, 431-436.

[13] D. H. Hyers, On the stability of the linear functional equation, Proc. Nat. Acad. Sci. U.S.A. 27 (1941), 222-224. 
[14] D. H. Hyers, G. Isac, and Th. M. Rassias, Stability of Functional Equations in Several Variables, Birkhäuser, Basel, 1998.

[15] G. Isac and Th. M. Rassias, Stability of $\psi$-additive mappings: Appications to nonlinear analysis, Internat. J. Math. Math. Sci. 19 (1996), no. 2, 219-228.

[16] K. Jun and H. Kim, The generalized Hyers-Ulam-Rassias stability of a cubic functional equation, J. Math. Anal. Appl. 274 (2002), no. 2, 267-278.

[17] S. Jung, Hyers-Ulam-Rassias Stability of Functional Equations in Mathematical Analysis, Hadronic Press lnc., Palm Harbor, Florida, 2001.

[18] A. K. Katsaras, Fuzzy topological vector spaces II, Fuzzy Sets and Systems 12 (1984), no. 2, 143-154.

[19] I. Kramosil and J. Michalek, Fuzzy metric and statistical metric spaces, Kybernetica 11 (1975), no. 5, 336-344.

[20] S. V. Krishna and K. K. M. Sarma, Separation of fuzzy normed linear spaces, Fuzzy Sets and Systems 63 (1994), no. 2, 207-217.

[21] S. Lee, S. Im, and I. Hwang, Quartic functional equations, J. Math. Anal. Appl. 307 (2005), no. 2, 387-394.

[22] D. Mihet and V. Radu, On the stability of the additive Cauchy functional equation in random normed spaces, J. Math. Anal. Appl. 343 (2008), no. 1, 567-572.

[23] M. Mirzavaziri and M. S. Moslehian, A fixed point approach to stability of a quadratic equation, Bull. Braz. Math. Soc. 37 (2006), no. 3, 361-376.

[24] A. K. Mirmostafaee, M. Mirzavaziri, and M. S. Moslehian, Fuzzy stability of the Jensen functional equation, Fuzzy Sets and Systems 159 (2008), no. 6, 730-738.

[25] A. K. Mirmostafaee and M. S. Moslehian, Fuzzy versions of Hyers-Ulam-Rassias theorem, Fuzzy Sets and Systems 159 (2008), no. 6, 720-729.

[26] _ Fuzzy approximately cubic mappings, Inform. Sci. 178 (2008), no. 19, 37913798.

[27] C. Park, Fixed points and Hyers-Ulam-Rassias stability of Cauchy-Jensen functional equations in Banach algebras, Fixed Point Theory Appl. 2007 (2007), Art. ID 50175.

[28] _ Generalized Hyers-Ulam stability of quadratic functional equations: a fixed point approach, Fixed Point Theory Appl. 2008 (2008), Art. ID 493751.

[29] C. Park, Y. Cho, and M. Han, Functional inequalities associated with Jordan-von Neumann type additive functional equations, J. Inequal. Appl. 2007 (2007), Art. ID 41820.

[30] C. Park and J. Cui, Generalized stability of $C^{*}$-ternary quadratic mappings, Abstr. Appl. Anal. 2007 (2007), Art. ID 23282.

[31] C. Park and A. Najati, Homomorphisms and derivations in $C^{*}$-algebras, Abstr. Appl. Anal. 2007 (2007), Art. ID 80630.

[32] V. Radu, The fixed point alternative and the stability of functional equations, Fixed Point Theory 4 (2003), no. 1, 91-96.

[33] Th. M. Rassias, On the stability of the linear mapping in Banach spaces, Proc. Amer. Math. Soc. 72 (1978), no. 2, 297-300.

[34] - On the stability of functional equations and a problem of Ulam, Acta Appl. Math. 62 (2000), no. 1, 23-130.

[35] F. Skof, Local properties and approximation of operators, Rend. Sem. Mat. Fis. Milano 53 (1983), 113-129.

[36] S. M. Ulam, A Collection of the Mathematical Problems, Interscience Publ. New York, 1960.

[37] J. Z. Xiao and X. H. Zhu, Fuzzy normed spaces of operators and its completeness, Fuzzy Sets and Systems 133 (2003), no. 3, 389-399. 
Sun-Young JANG

Department of Mathematics

UNIVERSITY OF ULSAN

Ulsan 680-749, Korea

E-mail address: jsym@ulsan.ac.kr

Choonkil Park

Department of Mathematics

HANYANG UNIVERSITY

SEOUL 133-791, KoREA

E-mail address: baak@hanyang.ac.kr

Dong Yun SHIN

Department of Mathematics

UNIVERSITY OF SEOUL

SEOUl 130-743, KoreA

E-mail address: dyshin@uos.ac.kr 